\title{
Normal filopodia extension in VASP-deficient platelets upon activation by adhesive matrices or soluble agonists
}

\author{
Sandra Eliautou'; Pierre Henri Mangin'; Ulrich Walter²; Christian Gachet'; François Lanza' \\ 'UMR_S 949 INSERM-Université de Strasbourg, Etablissement Français du Sang-Alsace, Strasbourg Cedex, France; ${ }^{2}$ Institute of Clinical \\ Biochemistry and Pathobiochemistry, University of Würzburg, Würzburg, Germany
}

\section{Dear Sir,}

Shape change is one of the earliest visible responses after platelet activation with a soluble agonist or following contact with an adhesive substratum (1). Platelet shape change is a composite of several distinct morphological changes. Early observations reported that ADP-activated platelets undergo transformation from a smooth discoid shape, to discs extending a few short pseudopodia, and progressively to spheres with numerous long filopodial extensions (1). The same types of transformations have since been observed also in response to adhesive matrices such as von Willebrand factor (VWF) (2) and fibrinogen (3). On these matrices progressive growth of lamellipodia finally results in extensive platelet spreading. Whereas filopodia and lamelli-

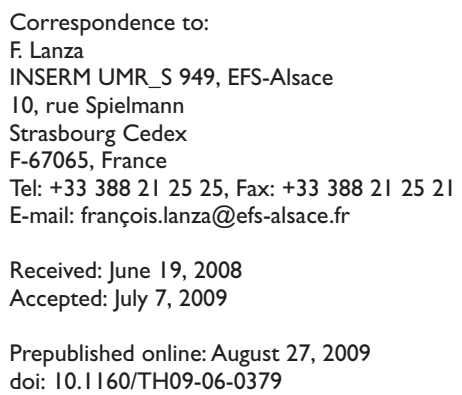

Thromb Haemost 2009; 102: 792-794 podia are membrane protrusions driven by actin polymerisation, sphering of the platelet body has been linked to a distinct process powered by an actomyosin-driven contraction, tubulin depolymerisation, and cortical actin cytoskeleton severing.

Despite being one of the earliest manifestations of platelet stimulation, the mechanisms governing filopodia formation and their role in platelet physiology and haemostasis are still largely unknown. A physiological role has been postulated for the ability of filopodia to multiply $\alpha_{\mathrm{IIb}} \beta_{3}$ fibrinogen contacts (4), thereby increasing platelet aggregation, as well as to slow-down platelet translocation under shear conditions, thus facilitating adhesion to the subendothelium (5). In motile and migrating cells, filopodia are proposed to participate in the migration process, in cell adhesion and exploring environmental cues (6). Filopodia are dynamic thin extensions made of parallel bundles of actin filaments. The mechanism of filopodia extension in platelets has been postulated to resemble that of other cell types, but in fact is still largely unknown. An important distinction between filopodia of adherent cells and those of platelets is that the latter does not require a pre-existing lamellipodial network and adherence to a substratum (7).

Among several filopodia regulatory proteins identified in other cell systems, vasodilator-stimulated phosphoprotein (VASP) has been proposed as an actin-binding positive modulator of filopodia extension (8). VASP belongs to the Ena/VASP family along with Mena and EVL (Ena-VASP-like), which is characterised by the presence of EVH1, EVH2 (Ena/VASP 
Figure I: Role of vasodilator-stimulated phosphoprotein (VASP) in platelet filopodia extension. Blood from wild-type and VASP knock-out (-/-) mice was drawn from the abdominal aorta into acid citrate dextrose (ACD) anticoagulant and a washed platelet suspension (300000 platelet/ $\mu$ l) was prepared in Tyrode's albumin buffer as described previously (20). (A) Platelets (a) were treated with $40 \mu \mathrm{g} / \mathrm{ml}$ Integrilin and I $\mu \mathrm{g} / \mathrm{ml}$ botrocetin and allowed to adhere for $20 \mathrm{~min}$ to cover slips coated with 10 $\mu \mathrm{g} / \mathrm{ml}$ of mouse von Willebrand factor (VWF), (b) were left untreated and allowed to adhere for $60 \mathrm{~min}$ on cover slips coated with $100 \mu \mathrm{g} / \mathrm{ml}$ of human fibrinogen (FBG). (B) Platelets treated with $40 \mu \mathrm{g} / \mathrm{ml}$ Integrilin were activated during $2 \mathrm{~min}$ in suspension without stirring by I $\mu$ M ADP, $200 \mu \mathrm{M}$ protease activated receptor 4 peptide (PAR-4) or I $\mu \mathrm{M}$ thromboxane $A 2$ analog U46619. All experiments were performed at $37^{\circ} \mathrm{C}$. Samples were fixed with $2,5 \%$ glutharaldehyde, processed for Scanning Electron Microscopy and analysed. Filopodia formation was scored according to the number of extensions per platelet ( 0 to I, 2 to 4,5 to 7 , $>7$ ) in five different fields corresponding to a total surface of $9215 \mu \mathrm{m}^{2}$. Bar represents 2,5 $\mu \mathrm{m}$. The results are mean values ( \pm SEM) from three separate experiments with 2 animals/experiment. The results are not statistically different using a Student's $t$ test. (a) VWF

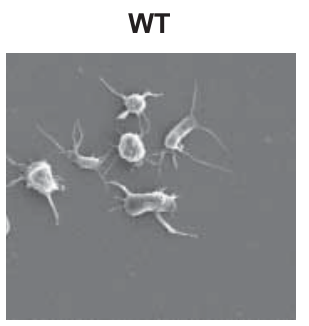

(b) FBG
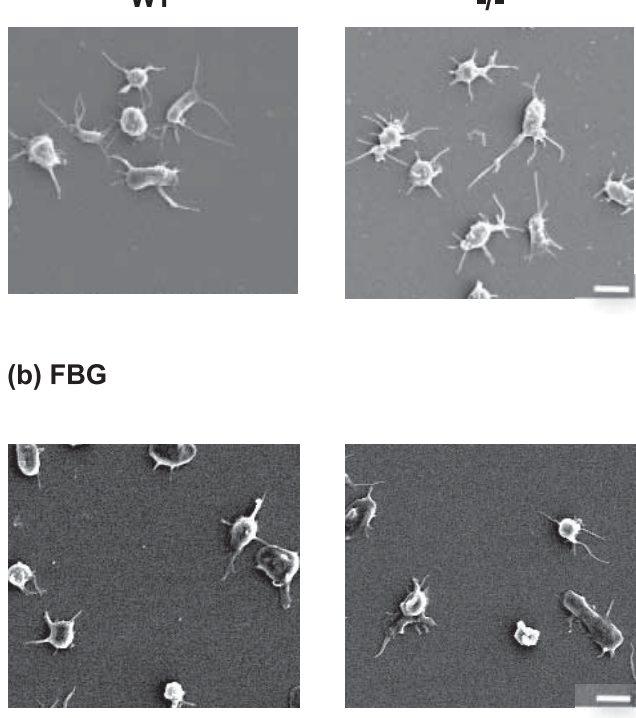

B
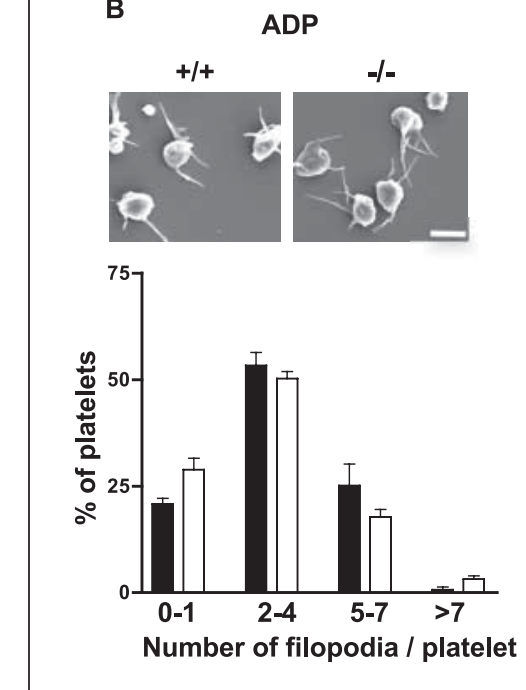
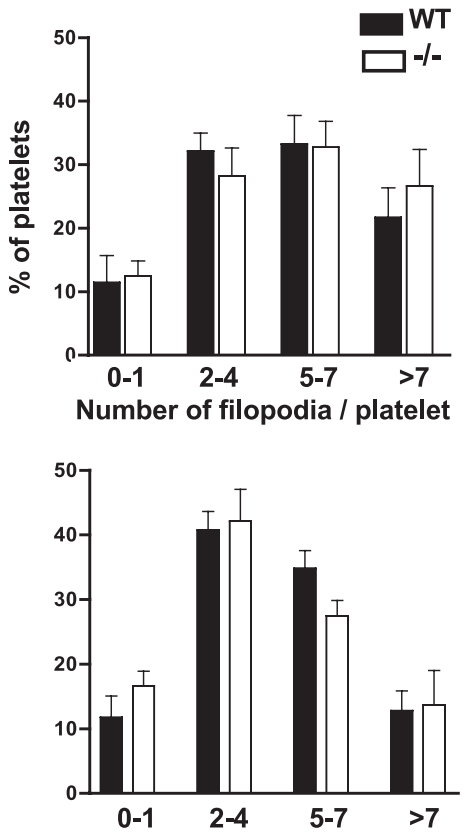

Number of filopodia / platele
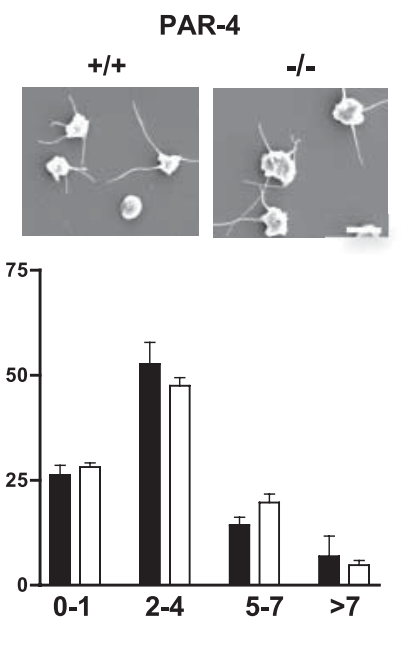
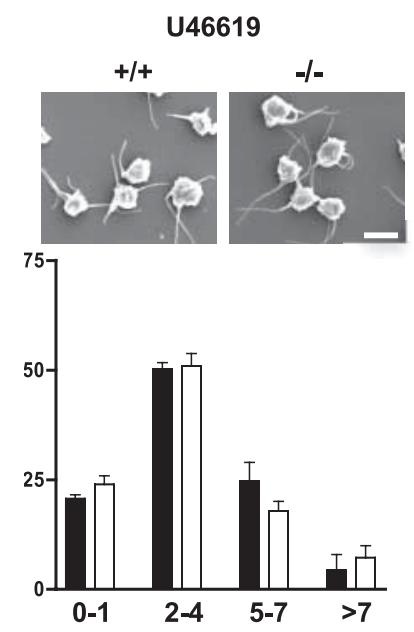

homology 1 and 2), and proline-rich central domains. The EVH2 domain contains a G- and F-actin-binding region (9) which contributes to the ability of VASP to regulate actin polymerisation with anti-capping, nucleation and bundling activities (10). The anti-capping activity is reinforced by the capacity of the VASP proline-rich domain to recruit profilin. Moreover, VASP interacts with proteins involved in filopodia extension and cytoskeletal dynamics such as src and Abl kinases (Abelson protein tyrosine kinase) (11).

VASP is phosphorylated by cAMP and cGMP-dependent protein kinases PKA and PKG resulting in decreased anti-capping and bundling activities $(12,13)$. Clinically, phosphorylated VASP is used as a marker to monitor the efficiency of treatments with the P2Y12 ADP receptor targeting drug clopidogrel (14, $15)$.

$\mathrm{VASP}^{-/}$mice have shown slight increases in in vitro platelet aggregation, secretion and fibrinogen-binding responses (16, 17). A more pronounced difference was noted under conditions in which cAMP and especially cGMP levels where increased. With VASP-deficient platelets, especially the cGMP-mediated inhibition of agonist-evoked platelet aggregation and FBG binding was impaired indicating a role of VASP as a negative modulator of integrin $\alpha_{\text {IIb }} \beta_{3}$ activation $(16,17)$. Also, the NO donor SNP (sodium-nitroprusside) induced inhibition of thrombusformation in flow-chambers with collagen-coated cover slips was strongly impaired with VASP KO platelets (18). In vivo, 
VASP-deficiency resulted in increased platelet adhesion to the injured vascular wall as well as impairment of the NO-induced inhibition using a model of arterial thrombosis (19).

In an attempt to better understand the mechanisms and role of filopodia formation in platelets, we investigated the consequences of VASP-deficiency on filopodia formation of platelets exposed to adhesive matrices (VWF and FBG) and soluble agonists (ADP, PAR-4 and the thromboxane A2 analogue U46619). Filopodia formation has been identified as a GPIb-dependent response of platelets adhering to a VWF matrix $(2,20)$. A majority of the mouse platelets adhering for 20 minutes on mouse VWF in the presence of botrocetin loose their discoid shape and extend filopodia independently of an RGD-integrin $\alpha_{\mathrm{IIb}} \beta_{3}$ mechanism. This response was not prevented in VASP ${ }^{-/-}$ mice in which, similar to wild type, $90 \%$ of adherent platelets extended one or more filopodia and exhibited a similar distribution in number of filopodia per platelet (Fig 1. A. a). Platelet adhesion to immobilised FBG also results in filopodia extension via an $\alpha_{\mathrm{II}} \beta_{3}$ dependent process. As observed for the VWF matrix, VASP deficiency did not modify the capacity of platelets to extend filopodia on FBG (Fig 1. A. b).

Filopodia formation was also analysed in platelets kept in suspension and activated by agonists acting on separate receptors (ADP, a PAR-4 agonist peptide and U46619). Under conditions in which aggregation was prevented (no stirring, no added FBG and presence of an $\alpha_{\mathrm{II}} \beta_{3}$ antagonist), a similar proportion of wild type and VASP-deficient platelets (75\% of total) exhibited filopodia with these different stimuli. Here also, a normal distribution was observed in $\mathrm{VASP}^{-/}$after scoring for the number of filopodia per platelet (Fig 1. B).

Altogether, the above results indicate that lack of VASP has little impact on filopodia formation after exposure to adhesive matrices or soluble agonists. In contrast, a previous study suggested a role of VASP downstream of PKCdelta mediating its negative effect on filopodia formation (21). The discrepancy could originate from differences in the type of matrix, the chronology and the nature of filopodia being analysed. Pula et al, had examined platelets adhering on a collagen matrix and at times where full spreading had occurred. Under these conditions, platelets with PKCdelta deficiency or blockade harboured a mixed morphology qualified as 'filopodia formation...superimposed upon a persistent lamellipodia formation in the fully spread pla- telet' which was absent in VASP deficient platelets. One hypothesis is that a separate event was evaluated here, whereby PKCdelta blockade engaged filopodia extension from existing lamellipodia, whereas the present work focused on filopodia formation prior to spreading. The response in the former study could resemble that documented in nucleated cells migrating on an adhesive matrix, which extend filopodia from preformed lamellipodia at their leading edge, and where a positive role of VASP has been postulated (22). Although this has not been formally documented in their study, the fact that VASP-deficient platelets normally spread on collagen in the absence of PKC delta blockade (21), suggests that the early filopodia transformation might also be normal on this matrix.

The normal filopodia response reported here in VASP deficiency contrasts with the increased FBG-binding, aggregation and secretion responses noted under conditions in which cAMP and cGMP levels where increased $(16,17)$. However, attempts to reveal a putative increase in filopodia formation in $\mathrm{VASP}^{-/-}$under similar conditions of increased cAMP and cGMP levels were obviated by the normal capacity of WT platelets to extend filopodia on VWF or FBG after pretreating with Sp-5,6-DCI-cBIMPS or 8-pCPT-cGMP, thus making it unlikely that VASP plays any role here (Supplementary Figure, available online at http://www. thrombosis-online.com). Normal filopodia extension under conditions of increased cAMP has been previously reported by Savage et al. in PGE1-treated platelets adhering to fibrinogen, supporting the present observation (23).

In conclusion, the present evaluation of $\mathrm{VASP}^{-/}$mice suggests that VASP is not essential in the early process of filopodia formation in platelets. Additional work is needed to clarify the mechanisms of filopodia formation and to test the in vivo importance of this platelet response in haemostasis. Despite the recognised value of the analysis of VASP phosphorylation in the surveillance of patients treated with the antiplatelet drug clopidogrel (15), the exact function(s) and importance of VASP and VASP phosphorylation in platelet function remain to be fully elucidated. Since VASP is an established substrate of cGMP and cAMP dependent kinases, and is involved in cyclic-nucleotide(and especially cGMP-) mediated inhibition of platelet adhesion (19) its role may be more significant under situations where these nucleotides are increased in response to prostacyclin and/ or nitric oxide.

\section{References}

1. Allen RD, Zacharski LR, Widirstky ST, et al. Transformation and motility of human platelets: details of the shape change and release reaction observed by optical and electron microscopy. J Cell Biol 1979; 83: 126-142

2. Yuan Y, Kulkarni S, Ulsemer P, et al. The von Willebrand factor-glycoprotein $\mathrm{Ib} / \mathrm{V} / \mathrm{IX}$ interaction induces actin polymerization and cytoskeletal reorganization in rolling platelets and glycoprotein $\mathrm{Ib} / \mathrm{V} / \mathrm{IX}$-transfected cells. J Biol Chem 1999; 274: 36241-36251.

3. Savage B, Ruggeri ZM. Selective recognition of adhesive sites in surface-bound fibrinogen by glycoprotein IIb-IIIa on nonactivated platelets. J Biol Chem 1991; 266: 11227-11233.
4. Hensler ME, Frojmovic M, Taylor RG, et al. Platelet morphologic changes and fibrinogen receptor localization. Initial responses in ADP-activated human platelets. Am J Pathol 1992; 141: 707-719.

5. Maxwell MJ, Dopheide SM, Turner SJ, et al. Shear induces a unique series of morphological changes in translocating platelets: effects of morphology on translocation dynamics. Arterioscler Thromb Vasc Biol 2006; 26: 663-669.

6. Faix J, Rottner K. The making of filopodia. Curr Opin Cell Biol 2006; 18: 18-25.

7. Chhabra ES, Higgs HN. The many faces of actin: matching assembly factors with cellular structures. Nat Cell Biol 2007; 9: 1110-1121.
8. Schirenbeck A, Arasada R, Bretschneider T, et al. The bundling activity of vasodilator-stimulated phosphoprotein is required for filopodium formation. Proceedings of the National Academy of Sciences of the United States of America. 2006; 103: 7694-7699.

9. Kwiatkowski AV, Gertler FB, Loureiro JJ. Function and regulation of Ena/VASP proteins. Trends Cell Biol 2003; 13: 386-392.

10. Krause M, Dent EW, Bear JE, et al. Ena/VASP proteins: regulators of the actin cytoskeleton and cell migration. Annu Rev Cell Dev Biol 2003; 19: 541-564. 11. Reinhard M, Jarchau T, Walter U. Actin-based motility: stop and go with Ena/VASP proteins. Trends Biochem Sci 2001; 26: 243-249. 
12. Barzik M, Kotova TI, Higgs HN, et al. Ena/VASP proteins enhance actin polymerization in the presence of barbed end capping proteins. J Biol Chem 2005; 280: 28653-28662.

13. Harbeck B, Huttelmaier S, Schluter K, et al. Phosphorylation of the vasodilator-stimulated phosphoprotein regulates its interaction with actin. J Biol Chem 2000; 275: 30817-30825.

14. Schwarz UR, Geiger J, Walter U, et al. Flow cytometry analysis of intracellular VASP phosphorylation for the assessment of activating and inhibitory signal transduction pathways in human platelets - definition and detection of ticlopidine/clopidogrel effects Thrombosis and haemostasis. 1999; 82: 1145-1152. 15. Aleil B, Ravanat C, Cazenave JP, et al. Flow cytometric analysis of intraplatelet VASP phosphorylation for the detection of clopidogrel resistance in patients with ischemic cardiovascular diseases. J Thromb Haemost. 2005; 3: 85-92.
16. Aszodi A, Pfeifer A, Ahmad M, et al. The vasodilator-stimulated phosphoprotein (VASP) is involved in cGMP- and cAMP-mediated inhibition of agonist-induced platelet aggregation, but is dispensable for smooth muscle function. Embo J 1999; 18: 37-48.

17. Hauser W, Knobeloch KP, Eigenthaler M, et al. Megakaryocyte hyperplasia and enhanced agonist-induced platelet activation in vasodilator-stimulated phosphoprotein knockout mice. Proc Natl Acad Sci U S A. 1999; 96: 8120-8125.

18. Begonja AJ, Teichmann L, Geiger J, et al. Platelet regulation by $\mathrm{NO} / \mathrm{cGMP}$ signaling and $\mathrm{NAD}(\mathrm{P}) \mathrm{H}$ oxidase-generated ROS. Blood cells, molecules \& diseases. 2006; 36: 166-170.

19. Massberg S, Gruner S, Konrad I, et al. Enhanced in vivo platelet adhesion in vasodilator-stimulated phosphoprotein (VASP)-deficient mice. Blood 2004; 103 : 136-142.
20. Mangin P, Yuan Y, Goncalves I, et al. Signaling role for phospholipase $\mathrm{C}$ gamma 2 in platelet glycoprotein Ib alpha calcium flux and cytoskeletal reorganization. Involvement of a pathway distinct from FcR gamma chain and Fc gamma RIIA. J Biol Chem 2003; 278: 32880-32891.

21. Pula G, Schuh K, Nakayama K, et al. PKCdelta regulates collagen-induced platelet aggregation through inhibition of VASP-mediated filopodia formation. Blood 2006; 108: 4035-4044.

22. Gupton SL, Gertler FB. Filopodia: the fingers that do the walking. Sci STKE. 2007 Sep 8; 2007(400): re5. 23. Savage B, Shattil SJ, Ruggeri ZM. Modulation of platelet function through adhesion receptors. A dual role for glycoprotein IIb-IIIa (integrin alpha IIb beta 3) mediated by fibrinogen and glycoprotein Ib-von Willebrand factor. J Biol Chem. 1992; 267: 11300-11306. 